\title{
Research on Product Lifecycle Management System for Household Appliance Based on RFID and EPC
}

\author{
Weigang Guo \\ School of Electronic and Information Engineering \\ Foshan University \\ Foshan, Guangdong Province, China \\ wgguo@qq.com \\ Jianqin Xie \\ School of Electronic and Information Engineering \\ Foshan University \\ Foshan, Guangdong Province, China \\ 34603770@qq.com
}

\author{
Dongyang Tu \\ University Library \\ Foshan University \\ Foshan, Guangdong Province, China \\ 88059994@qq.com
}

\begin{abstract}
For fulfilling the household appliance's product lifecycle management, in this paper, while taking the refrigerator as the research object, a product lifecycle management system based on radio frequency identification and the electronic product code internet of things is designed and developed. The PLM system has five subsystems: production subsystem, warehousing and logistics subsystem, sales subsystem, maintenance subsystem, recycling subsystem. The techniques of the RFID labels and EPC encoding, object name service, electronic product code information service, RFID middleware are detailed discussed and developed. The system can be used to fulfill the household appliance's whole PLM process including warehousing, logistics and distribution, sales, repair, recycling and product tracking and tracing. The system has been applied in a refrigerator manufacturing enterprise in Guangdong, China. It improves the accuracy of product information acquisition and transmission, also the logistics efficiency, and reduces the cost, provides better service for the consumer. The system has a good prospect of application and extension.
\end{abstract}

Keywords-household appliance; product lifecycle management system; internet of things; EPC; RFID

\section{INTRODUCTION}

RFID technologies in product lifecycle management in western countries have been widely used. Many high value goods, such as cars, copiers have been embed RFID tags to strengthen product management and service, and have achieved good benefits $[1,2]$. In China, there are also a few researches on RFID and PLM combination. Cai proposed a solution of the whole lifecycle household appliance's monitoring and tracking system based on RFID technology [3]. And, based on the working procedures of used household electric appliance management in China, Bing presented an information management system suitable for China, which employed RFID technology [4] $\mathrm{Ji}$ introduced and applied the IOT technology to the quality monitoring and management of the whole process to supervise the status of the tobacco leaves in their life circle and make quick response to it [5].QIN designed the closed-loop product lifecycle management system based on the IOT technology from the perspective of enterprise application system integration and commercial mode innovation [6].

From the above literatures it can be seen that for the study of the application of RFID in household appliance, although the domestic experts and scholars have made some achievements in some aspects, but have yet to achieved full lifecycle management. This paper takes the refrigerator as the research object, then designs and develops a whole product lifecycle management (PLM) based on RFID and EPC.

\section{THE OVERALL STRUCTURE OF THE SYSTEM}

The PLM system mainly consists of a EPC code management scheme, ONS server, EPCIS server, database server. The overall structure of the system is shown in Fig .1. The system adopts the EPC specification proposed by EPCglobal. Each node enterprise has its own EPCIS server in which stores EPCIS event data. The local ONS (object name service) server stores the EPC codes and their mapping information to the address of the EPCIS server [7]. At the same time, enterprise also needs to store EPC codes and their mapping information to the local ONS server address into the system root ONS server. 


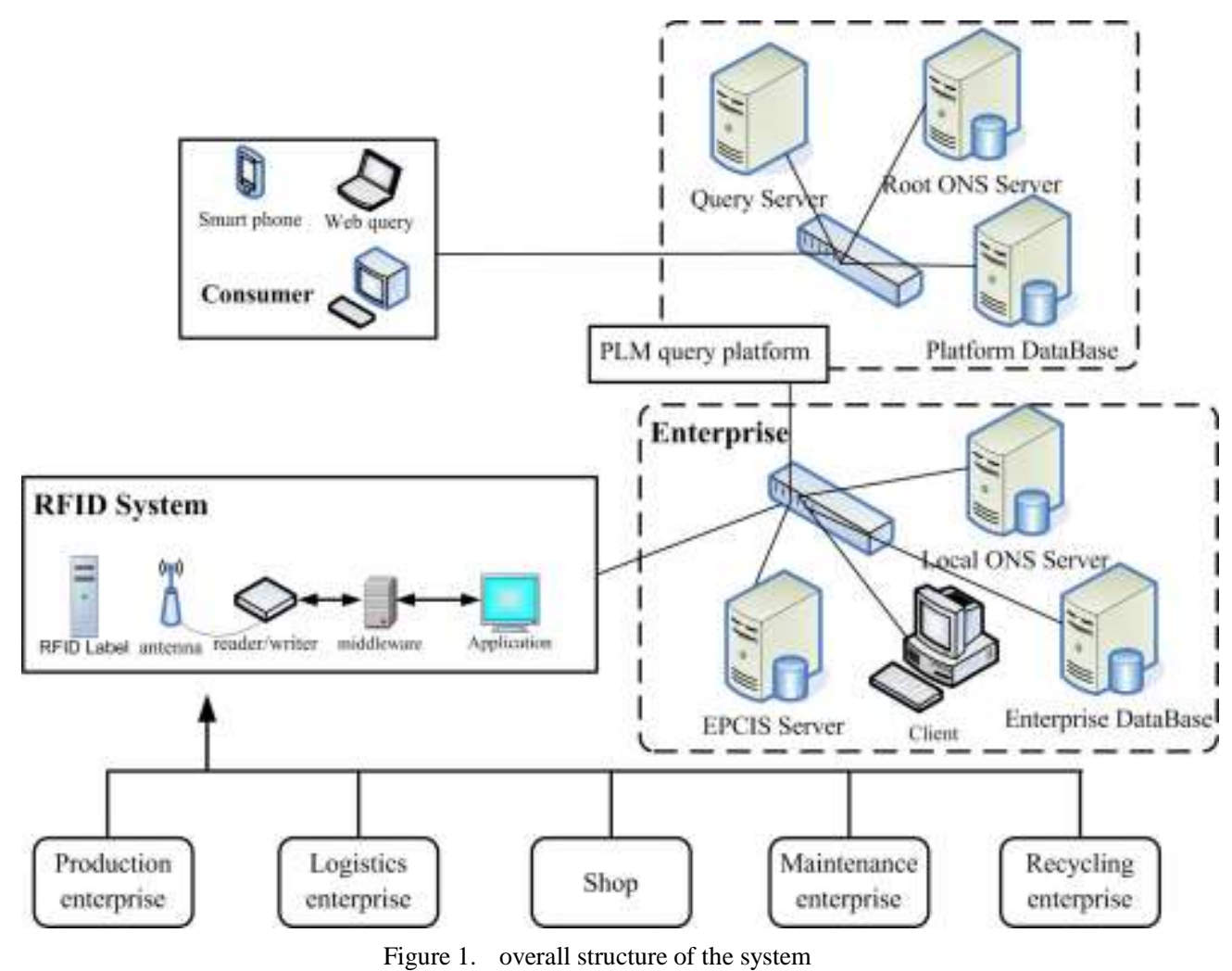

In the refrigerator production enterprises, while the refrigerator is produced and passed inspection, each product is assigned an EPC code and written to the RFID label which is stuck on the refrigerator. Product information is written to the database by the product manager.

In the process of circulation and daily use, logistics enterprises, and sales enterprise, maintenance enterprises, recycling enterprises access product data by using the reader and writer device and save it to its own EPCIS server, and register the EPCIS server address on the local

ONS server, and register the local ONS server address on the root ONS server.

When Consumer inquiries, enterprise's local ONS server address is obtained through the root ONS server, and then access the EPCIS server through the firm's local ONS server and obtain product information.

Fig .2 shows the system level of PLM. The system level includes hardware layer which provides data collection services, service layer which provides the data processing, data layer which provides information storage and the application layer which provides a variety of business application.

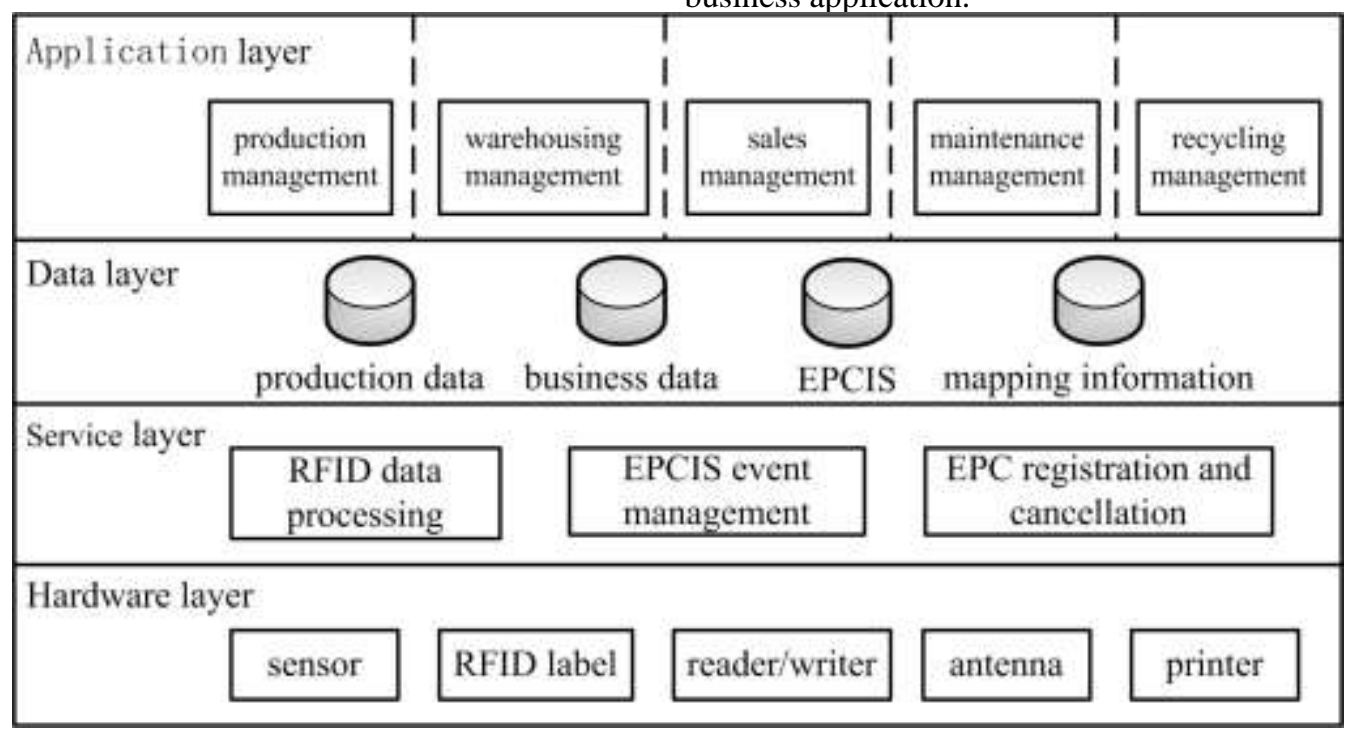

Figure 2. the system level of PLM 


\begin{tabular}{|c|c|c|c|c|}
\hline Type & Header & General Manager Number & Object Class & Serial Number \\
\hline GID-96 & 8 & 28 & 24 & 36 \\
\hline & & the reserved field 20, company ID 8 & $\begin{array}{c}\text { The reserved field 8, product sales } \\
\text { destination 6, product series 10 }\end{array}$ & $\begin{array}{c}\text { Production date 16, product } \\
\text { serial number 20 }\end{array}$ \\
\hline
\end{tabular}

\section{DESIGN OF RFID LABEL}

EPC code is used through the product from raw material production, processing, storage and sales throughout the product lifecycle. It is the best support for efficient monitoring and product management.

\section{A. Household appliance RFID label's EPC encoding type Selecting a Template}

EPC code data standard GID-96 $[8,9]$ is selected as the household appliance products code. The General Identifier is defined for a 96-bit EPC, and is independent of any existing identity specification or convention. The General Identifier is composed of three fields -the General Manager Number (28 bits), Object Class (24 bits) and Serial Number (36 bits). Encodings of the GID include a fourth field, the header ( 8 bits), to guarantee uniqueness in the EPC namespace.

\section{B. EPC encoding method}

According to the coding rules and code structure of encoding system, code distribution should be under the premise of ensuring capacity, as careful as possible to identify the identity of the object. Combined with the survey of household appliance enterprises production characteristics and various products in the market, the EPC encoding regions are designed and distributed. The encoding target is to achieve the uniqueness, permanent, simplicity and scalability features. The detailed encoding method is shown in Table I.

\section{ONS DESIGN}

The ONS design of PLM system mainly includes ONS server constructing, EPC registration and cancellation; ONS query process and algorithm, ONS parsing process [10].

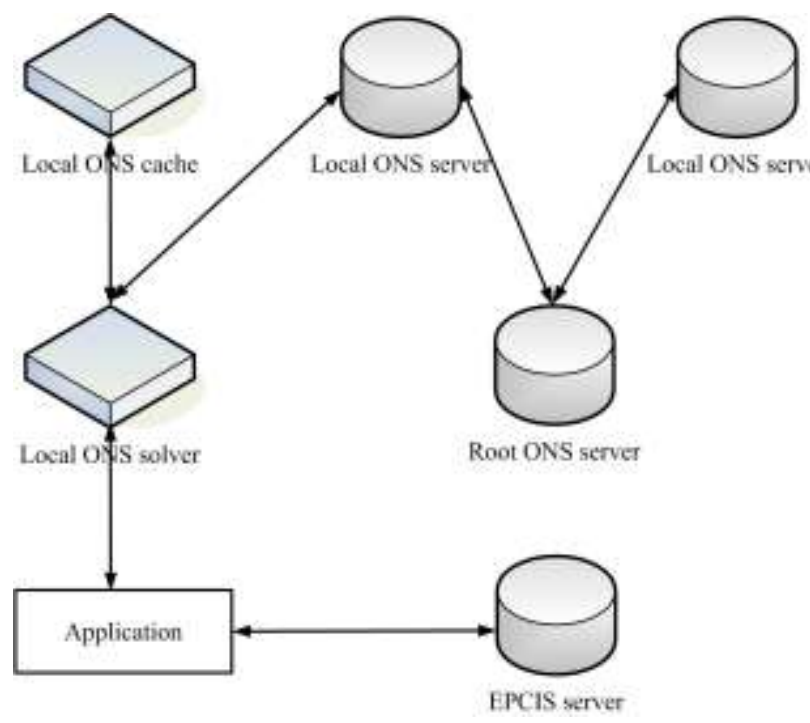

Figure 3. the system architecture of ONS
ONS provides a global query service. The service puts a EPC code into one or more NAPTR (naming authority pointer) records, and then obtain the address of the EPCIS server through NAPTR records, so as to obtain detailed information of the products.

The ONS designed in this paper of adopts the $\mathrm{B} / \mathrm{S}$ model. It is hierarchical, distributed system architecture. The ONS system consists of mapping information, the root ONS server, the local ONS server, a local ONS cache, and local ONS solver. The ONS system architecture is shown in Fig .3.

The EPC registration is the initial step of products into the Internet of things. To achieve the purpose of sharing information, EPC should be registered to the ONS. When EPC is deleted, it should be cancelled from the ONS in order to ensure the accuracy of the information.

The ONS query algorithm mainly includes 3 parts: EPC encoding module on the local server, a local ONS solver of GID-URI into the DNS module and the local ONS solver's query sending module.

ONS parsing services are constructed on the DNS architecture. It's parsing process is the same as DNS. ONS client can send parsing request directly to the ONS server, and then the ONS server returns the parsing result.

\section{EPC INFORMATION SERVICE}

EPCIS information services include EPCIS event definition, EPCIS event capture and EPCIS event query [11]. The EPCIS event definition is the key to the successful realization of the PLM system.

EPCIS event definition: According to the EPCIS standard released by EPCglobal, There are four EPCIS events: Object Event, Aggregation Event, Quantity Event, and Transaction Event. Throughout the life cycle of household appliances from the production of the factory to recycling, there are 8 typical EPCIS events, as shown in Table II. While the system is in the process of business, the involved EPCIS events should be uploaded to a EPCIS server in accordance with the agreed manner.

TABLE II.

EPCIS EVENT DEFINITION

\begin{tabular}{|l|c|l|l|}
\hline \multicolumn{1}{|c|}{ Business link } & Number & \multicolumn{1}{c|}{ Name } & \multicolumn{1}{c|}{ Event type } \\
\hline Production & 1 & Product offline & Object Event \\
\hline \multirow{2}{*}{$\begin{array}{l}\text { Warehousing and } \\
\text { logistics }\end{array}$} & 2 & Product storage & Quantity Event \\
\cline { 2 - 4 } & 4 & Put in storage & $\begin{array}{l}\text { Transaction } \\
\text { Event }\end{array}$ \\
\hline \multirow{2}{*}{ Sales } & 5 & $\begin{array}{l}\text { Delivery from } \\
\text { storage }\end{array}$ & $\begin{array}{l}\text { Transaction } \\
\text { Event }\end{array}$ \\
\hline Maintenance & 6 & Sold & $\begin{array}{l}\text { Transaction } \\
\text { Event }\end{array}$ \\
\hline Recycling & 7 & Repair & $\begin{array}{l}\text { Agject Event } \\
\text { Event }\end{array}$ \\
\hline
\end{tabular}

EPCIS event capture: In the PLM system, the interfaces of event capture and query EPCIS server provided outwards are provided by Web Service. Event 
capture interface contains a simple method. That is to upload events in the form of XML.

EPCIS event query: The PLM system release the specified query mode using Web Service. The provided query mode is to enter a EPC code, and then return all the events containing the EPC code in time sequence.

\section{RFID MIDDLEWARE}

Functions of RFID middleware are not only transferring messages, but also including data security, data interpretation, network management, error controlling, hardware differences shielding, providing the standard unified data interface to upper layer, having good adaptability and security [12, 13]. RFID middleware subsystem mainly consists of the following parts:

System management: system management module provides the system login, exit the system, increase, delete, search and other operations.

Device management: This module mainly carries on the centralized management and configuration to physical reading/writing devices, including enabling and disabling the devices etc.

Data filtering: embedded RFID middleware receives a large collection of data from the reader. There are a lot of redundant information and some wrong information in the data. So, in order to transmit useful and right information to the application or superior middleware, filtering data and elimination of redundant data are the necessary functions.

Data analysis and summary: the original RFID data stream received from the reader/writer are simple and scattered information. In order to provide meaningful information to the application or other RFID middleware, it is necessary that for RFID data to do processing of aggregations and gets meaningful event information;

Information interaction: After filtering and aggregation process, RFID data will be transferred to the management system. XML data will be generated in accordance with the standard data format.

\section{CONCLUSIONS}

Household appliance products Lifecycle management system based on RFID and EPC is designed and developed in this paper. By using EPC specification proposed by EPCglobal and RFID label, the refrigerators are identified. And then it is realized that all link information throughout the lifecycle of the production plant, warehousing logistics distribution, sales, repair, and product recycling are managed. This research provides helpful references for other equipment such as elevators, automotive while developing lifecycle management. The main problems existing in this paper is the whole management process needs RFID reader, the convenience of operation is to be improved.

\section{ACKNOWLEDGMENT}

This work is completed under the support of the Foshan science and technology development project funds (No.2011AA100061).

\section{REFERENCES}

[1] S. F. Wamba, "A literature review of RFID-enabled healthcare applications and issues, "International Journal of Information Management. Vol.33,May 2013,pp:875-891.

[2] R. Shi and Z. Yang, Security Technology of IOT, Publishing House of Electronics Industry,Beijing,2013.

[3] X. Cai, D. Luo, and W. Zheng, "Design and Implementation of Household Appliance's Monitoring and Tracking System," Computer Technology and Development. Vol 21,Oct.2011, pp.176-178.

[4] X. Bing, P. Zhao, "Research and Development of Information Management System for Used Household Electric Appliance Based on RFID Technology," China Appliance Technology. May 2007, pp.45-48.

[5] X. Ji, M. Jiang, and X. Wu, "Study on Tobacco Leaves Life Circe Management System Based on IOT," Logistics Technology. Vol.32,Nov.2013,pp.399-401.doi:10.3969/j.issn.1005152X.2013.11.127.

[6] X. Qin, "Study on Integrated Closed-loop Product Life Cycle Management Based on IOT," Logistics Technology. Vol.31,Dec. 2012, pp.83-85, doi:10.3969/j.issn.1005-152X.2012.12.029.

[7] Dieter Uckelmann, Mark Harrison, Florian Michahelles, Architecting the Internet of Things,Springer-Verlag, Berlin Heidelberg,2011.

[8] GS1 EPCIS Standards on http://www.gs1.org/epcis/latest.

[9] S. Zhang, S. Li, and H. Huang, "The research and application of laoganma enterprise internet of RFID tag coding scheme based on EPC GID-96," Manufacturing Automation. Vol. 36,Sept.2014, pp.134-138, Doi : 10.3969/j.issn.1009-0134.2014.09.36.

[10] X. Dong, B. Yan, "Object Naming Services in the EPC Network," Microelectronics \& Computer. Vol.22, Feb.2005, pp.17-21.

[11] F. Li, "Analysis of ONS architecture and security in EPC internet of things," Netinfo Security.Dec.2010,pp.6-9.,doi 10.3969/j.issn.1671-1122.2010.12.003.

[12] H. Fu, "Related issues in EPC network middleware of enterprise information interaction," Computer Knowledge and Technology. Vol.6,March 2010, pp.2047-2048.

[13] Y. Zhang, F. Zhang, "Research on agriculture products traceability algorithms based on Internet of Things," Electronic Design Engineering.Vol.20, Aug.2012, pp.5-9. 